\title{
Influence of finite volume and magnetic field effects on the QCD phase diagram
}

\author{
Niseem Magdy, $1, *$ M.Csanád, ${ }^{1,2,+\dagger}$ and Roy.A.Lacey ${ }^{1,+}$ \\ ${ }^{1}$ Department of Chemistry, State University of New York, \\ Stony Brook, New York 11794, USA \\ ${ }^{2}$ Eötvös University, Department of Atomic Physics, H-1117 Budapest, Hungary
}

\begin{abstract}
The $2+1 S U(3)$ Polyakov linear sigma model (PLSM) is used to investigate the respective influence of a finite volume and a magnetic field on the quark-hadron phase boundary in the plane of baryon chemical potential $\left(\mu_{B}\right)$ vs. temperature $(T)$ of the QCD phase diagram. The calculated results indicate sizable shifts of the quark-hadron phase boundary to lower values of $\left(\mu_{B}\right.$ and $\left.T\right)$ for increasing magnetic field strength, and an opposite shift to higher values of $\left(\mu_{B}\right.$ and $\left.T\right)$ for decreasing system volume. Such shifts could have important implications for extraction of the thermodynamic properties of the QCD phase diagram from heavy ion data.

PACS numbers: 12.39.Fe, 12.38.Aw, 12.38.Mh
\end{abstract}

Keywords: Chiral Lagrangian, Quark confinement, Quark-Gluon Plasma

\footnotetext{
*niseem.abdelrahman@stonybrook.edu

$\dagger$ csanad@elte.hu

†roy.lacey@stonybrook.edu
} 


\section{INTRODUCTION}

A major impetus for current heavy ion research is the prospect of obtaining profound insights on the rich phase structure of strongly interacting matter at high temperature and non-zero net baryon number density. Ongoing programs at RHIC [1], the SPS [2] and the LHC [3], as well as future facilities at FAIR [4] and NICA [5] are at the forefront of experimental efforts designed to map the thermodynamic and transport properties of this strongly interacting QCD matter. Lattice QCD simulations suggests a smooth cross over phase transition from hadronic matter to the quark gluon phase at low density and high temperature [6, 7]. At high density and low temperature a first order phase transition is expected [8 13]. Both transition domains, crossover and the first-order phase-transition, are connected by the expected critical endpoint (CEP), at which the phase transition is likely second order. The beam energy scan (BES) program at RHIC, have begun to show striking non-monotoic signatures which could be an indication that the CEP is located at high temperature and modest values of baryon chemical potential $\left(\mu_{B}\right)$ [14].

A preponderance of the theoretical studies assume an infinite volume devoid of magnetic fields, for the QCD matter produced in heavy ion collisions. This is in stark contrast to the finite volumes and sizable magnetic fields produced in these collisions (both depend on the size of the colliding nuclei, the center of mass energy $\left(\sqrt{s_{N N}}\right)$ and the collision centrality). Therefore, it is important to ask whether the combined influence of a finite volume and a strong magnetic field leads to a modification of the apparent thermodynamic properties of the produced QCD medium.

The influence of a finite-volume and the presence of a strong magnetic field $(B)$ has been widely discussed in the literature [15-38]. This includes the effects on the value of the critical temperature, the location of the critical end point and other thermodynamic properties. Initial studies of the magnetic field effect include lattice QCD (lQCD) studies [23], the MIT bag model [15], the Nambu-Jona Lasinio (NJL) model [16, 17] and the Linear Sigma Model (LSM), or Quark Meson model (QM) [18 20], as well as extensions of the NJL and the LSM involving the Polyakov loop (PNJL and PLSM) [21, 22]. The results from these studies, which indicate an increase of the transition temperature $T_{c}$ with increasing magnetic

field [24], contrast with the results from recent lQCD calculations (with a physical pion mass $\left.m_{\pi}=140 \mathrm{MeV}\right)$ which indicate that $T_{c}$ decreases with increasing magnetic field. The PLSM 
and PNJL models have been recently used to study the latter trend for vanishing chemical potential [37, 39]. The effects of a finite volume [25, 28] which include a strong influence on the value of the transition temperature $\left(T_{c}\right)$, the location of the critical end point and other thermodynamic properties, have been extensively studied with the NJL [26], LSM [27, 38] and PNJL [29] models. However, strikingly different trends for the influence of finite-size effects on $T_{c}$ have been reported for the LSM and PNJL models.

In this work, we use the $2+1 S U(3)$ Polyakov Linear Sigma Model (PLSM) [40 42] with the Fukushima Polyakov loop effective potential [43] to investigate the combined effects of finite volumes and magnetic fields at temperatures and chemical potentials akin to those produced in heavy ion collisions over a broad range of beam collision energies. The present work is organized as follows. In section [1] we give a brief overview of the PLSM [37, 40], as well as the parameters of the model employed in this study. We then present the results of our study on the influence of finite-volume and the magnetic field effects on the PLSM order parameters (chiral condensates $\sigma_{x}$ and $\sigma_{y}$ and Polyakov loops $\phi$ and $\phi^{*}$ ), thermodynamic properties and the chiral phase transition in section III. We conclude with a summary and an outlook in section IV]

\section{THE POLYAKOV LINEAR SIGMA MODEL (PLSM)}

The $S U(3)$ Linear Sigma Model with $N_{f}=2+1$ flavor quarks, can be coupled to Polyakov loop dynamics to formulate the PLSM [40 42]. The associated Lagrangian is given as;

$$
\mathcal{L}=\mathcal{L}_{\text {chiral }}-\mathcal{U}\left(\phi, \phi^{*}, T\right)
$$

where the chiral part [quark $q$ and meson $m$ ] of the Lagrangian, $\mathcal{L}_{\text {chiral }}=\mathcal{L}_{q}+\mathcal{L}_{m}$, has $S U(3)_{L} \times S U(3)_{R}$ symmetry [44, 45]; $\mathcal{U}\left(\phi, \phi^{*}, T\right)$ represents the Polyakov loop effective potential [43]. This effective potential leads to reasonable agreement with recent lQCD results [46]. Other Polyakov loop potentials [47, 48] were also considered. However, the particular choice made for this work does not influence the main conclusions of our work.

$$
\mathcal{U}\left(\phi, \phi^{*}, T\right)=-b T\left\{54 \exp \left(-\frac{a}{T}\right) \phi \phi^{*}+\ln \left[1-6\left(\phi \phi^{*}\right)^{2}-3\left(\phi \phi^{*}\right)^{3}+4\left(\phi^{3}+\phi^{* 3}\right)\right]\right\},
$$

with $a=0.664 \mathrm{GeV}$ and $b=0.0075 \mathrm{GeV}^{3}[43$. The mean field approximation is used to obtain the grand potential [40] as; 


$$
\Omega\left(T, e B, \mu_{f}\right)=U\left(\sigma_{x}, \sigma_{y}\right)+\mathcal{U}\left(\phi, \phi^{*}, T\right)+\Omega_{\bar{\psi} \psi}\left(T, \mu_{f} ; \phi, \phi^{*}, B\right)+\delta_{0, B} \Omega_{\bar{\psi} \psi}^{0}\left(T, \mu_{f} ; \phi, \phi^{*}\right),(3)
$$

where the first term in Eq. (3) is a purely mesonic potential expressed as,

$$
\begin{aligned}
U\left(\sigma_{x}, \sigma_{y}\right) & =\frac{m^{2}}{2}\left(\sigma_{x}^{2}+\sigma_{y}^{2}\right)-h_{x} \sigma_{x}-h_{y} \sigma_{y}-\frac{c}{2 \sqrt{2}} \sigma_{x}^{2} \sigma_{y} \\
& +\frac{\lambda_{1}}{2} \sigma_{x}^{2} \sigma_{y}^{2}+\frac{1}{8}\left(2 \lambda_{1}+\lambda_{2}\right) \sigma_{x}^{4}+\frac{1}{4}\left(\lambda_{1}+\lambda_{2}\right) \sigma_{y}^{4}
\end{aligned}
$$

Here, $m^{2}, h_{x}, h_{y}, \lambda_{1}, \lambda_{2}$ and $c$ are model parameters as outlined in Ref. [44]. The values for these parameters, used in the present study, are tabulated in Table I below. Recent studies [49, 50] suggest that better consistency with recent lattice results can be achieved in the $T<T_{c}$ region, by extending the model within the vector meson sector. Such an extension was not included in this work and is not expected to strongly affect the qualitative conclusion.

The third term in Eq. (3) $\Omega_{\bar{\psi} \psi}\left(T, \mu_{f} ; \phi, \phi^{*}, B\right)$ represents the contributions from quarks and anti-quarks at a non-vanishing magnetic field strength. Using Landau quantization and magnetic catalysis concepts, this potential can be expressed as [37];

$$
\begin{aligned}
\Omega_{\bar{\psi} \psi}\left(T, \mu_{f} ; \phi, \phi^{*}, B\right)= & -2 \sum_{f} \frac{\left|q_{f}\right| B T}{2 \pi} \sum_{\nu=0}^{\infty} \int \frac{d p}{2 \pi}\left(2-1 \delta_{0 n}\right) \\
& \left\{\operatorname { l n } \left[1+3\left(\phi+\phi^{*} e^{\left.-\frac{\left(E_{f}^{\nu}-\mu_{f}\right)}{T}\right)} e^{-\frac{\left(E_{f}^{\nu}-\mu_{f}\right)}{T}}+e^{\left.-3 \frac{\left(E_{f}^{\nu}-\mu_{f}\right)}{T}\right]}\right.\right.\right. \\
& \left.+\ln \left[1+3\left(\phi^{*}+\phi e^{-\frac{\left(E_{f}^{\nu}+\mu_{f}\right)}{T}}\right) e^{-\frac{\left(E_{f}^{\nu}+\mu_{f}\right)}{T}}+e^{-3 \frac{\left(E_{f}^{\nu}+\mu_{f}\right)}{T}}\right]\right\},
\end{aligned}
$$

where $E_{f}^{\nu}$ is the modified quark dispersion [37] and $\mu_{f}$ denotes the quark chemical potentials. The subscript $f$ runs over different quark flavors. The quark chemical potentials are related to the baryon $\left(\mu_{B}\right)$, strange $\left(\mu_{S}\right)$ and charge $\left(\mu_{Q}\right)$ chemical potentials via the following transformations [51];

$$
\begin{aligned}
& \mu_{u}=\frac{\mu_{B}}{3}+\frac{2 \mu_{Q}}{3} \\
& \mu_{d}=\frac{\mu_{B}}{3}-\frac{\mu_{Q}}{3} \\
& \mu_{s}=\frac{\mu_{B}}{3}-\frac{\mu_{Q}}{3}-\mu_{S}
\end{aligned}
$$



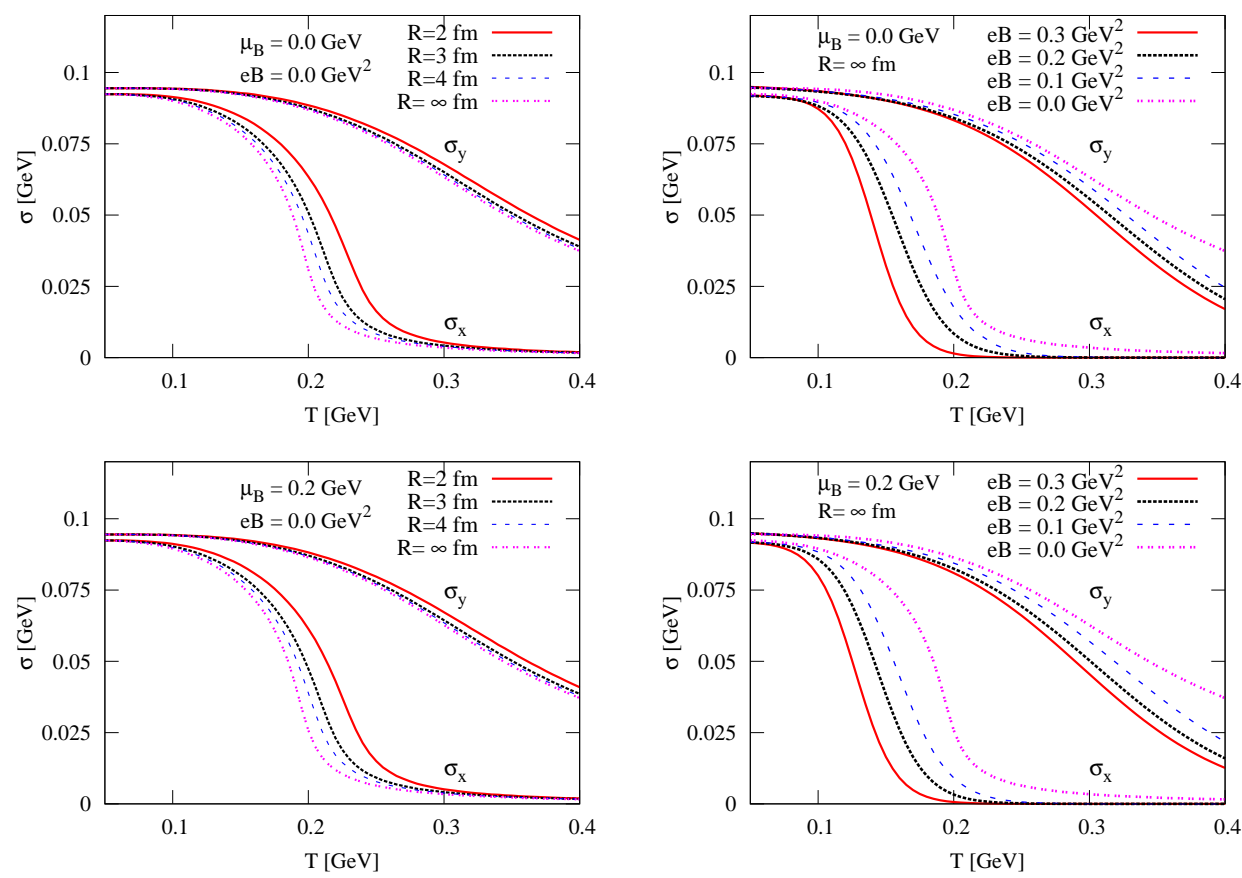

Fig. 1. (Color online) Temperature dependence of the chiral condensates $\sigma_{x}$ and $\sigma_{y}$, for several volume selections with $e B=0$ (left panels) and for several $e B$ selections at infinite volume (right panels). The results are shown for $\mu_{B}=0 \mathrm{GeV}$ (top panels) and $\mu_{B}=0.2 \mathrm{GeV}$ (bottom panels). A fit function is used for $e B>0$ results.

and $E_{f}^{\nu}$ is given as [37]:

$$
\begin{aligned}
& E_{u}^{\nu}=\sqrt{p_{z}^{2}+m_{q}^{2}+\left|q_{u}\right|(2 n+1-\sigma) B} \\
& E_{d}^{\nu}=\sqrt{p_{z}^{2}+m_{q}^{2}+\left|q_{d}\right|(2 n+1-\sigma) B} \\
& E_{s}^{\nu}=\sqrt{p_{z}^{2}+m_{s}^{2}+\left|q_{s}\right|(2 n+1-\sigma) B},
\end{aligned}
$$

where $\sigma$ is related to the spin quantum number $S(\sigma= \pm S / 2)$. Here, we have replaced $2 n+1-\sigma$ by one quantum number $\nu$, where $\nu=0$ is the Lowest Landau Level $m_{f}$, and $f$ runs over the $u, d$ and $s$ quark masses,

$$
\begin{aligned}
& m_{q}=g \frac{\sigma_{x}}{2} \\
& m_{s}=g \frac{\sigma_{y}}{\sqrt{2}} .
\end{aligned}
$$

The fourth term in Eq. (3) $\Omega_{\bar{\psi} \psi}^{0}\left(T, \mu_{f} ; \phi, \phi^{*}\right)$ gives the quark and anti-quark contributions 
for vanishing magnetic field. This potential can be expressed as [42],

$$
\begin{aligned}
& \Omega_{\bar{\psi} \psi}^{0}\left(T, \mu_{f} ; \phi, \phi^{*}\right)=-2 T \sum_{f} \int \frac{d^{3} p}{(2 \pi)^{3}} \\
& \left\{\ln \left[1+3\left(\phi+\phi^{*} e^{-\frac{\left(E_{f}^{0}-\mu_{f}\right)}{T}}\right) e^{-\frac{\left(E_{f}^{0}-\mu_{f}\right)}{T}}+e^{-3 \frac{\left(E_{f}^{0}-\mu_{f}\right)}{T}}\right]\right. \\
& \left.+\ln \left[1+3\left(\phi^{*}+\phi e^{-\frac{\left(E_{f}^{0}+\mu_{f}\right)}{T}}\right) e^{-\frac{\left(E_{f}^{0}+\mu_{f}\right)}{T}}+e^{-3 \frac{\left(E_{f}^{0}+\mu_{f}\right)}{T}}\right]\right\} \text {. }
\end{aligned}
$$

The effects of a finite volume are introduced in the PLSM via a lower momentum cut-off $p_{\text {min }}[G e V]=\pi / R[G e V]=\lambda$, where $R$ is the length of a cubic volume [52].

\section{RESULTS AND DISCUSSION}

In the following, several results are presented to illustrate the effects of finite volumes and magnetic field strengths on the PLSM order parameters, thermodynamic properties and the chiral phase transition. These results were all obtained with the values for the model

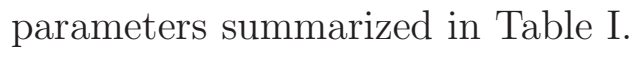

\begin{tabular}{|c|c|c|c|c|c|c|}
\hline$m_{\sigma}(\mathrm{MeV})$ & $c(\mathrm{MeV})$ & $\lambda_{1}$ & $m^{2}\left(\mathrm{MeV}^{2}\right)$ & $\lambda_{2}$ & $h_{x}\left(\mathrm{MeV}^{3}\right)$ & $h_{y}\left(\mathrm{MeV}^{3}\right)$ \\
\hline 800 & 4807.84 & 13.49 & $-(306.26)^{2}$ & 46.48 & $(120.73)^{3}$ & $(336.41)^{3}$ \\
\hline
\end{tabular}

Tab. I. Summary of the values of the PLSM parameters employed in the calculations. A detailed description of these parameters is given in Ref. [53].

\section{A. Order parameters}

Figure 1 shows the temperature dependence of the two chiral condensates $\left(\sigma_{y}\right.$ and $\left.\sigma_{x}\right)$ for different volume and magnetic field selections for two values of $\mu_{B}$. The left panels show that both chiral condensates increase as the system volume is decreased, albeit with much larger sensitivity for the non-strange chiral condensates $\left(\sigma_{x}\right)$. The right panels indicate an opposite trend for increasing magnetic field strength, again with with a larger sensitivity for $\sigma_{x}$. Fig. 2 shows the corresponding temperature dependence of the two Polyakov loops $(\phi$ and $\left.\phi^{*}\right)$ for the same volume, magnetic field and $\mu_{B}$ selections. For $\mu_{B}=0 \mathrm{GeV}$ we observe that $\phi=\phi^{*}$ and both order parametes show very little, if any, dependence on the volume 

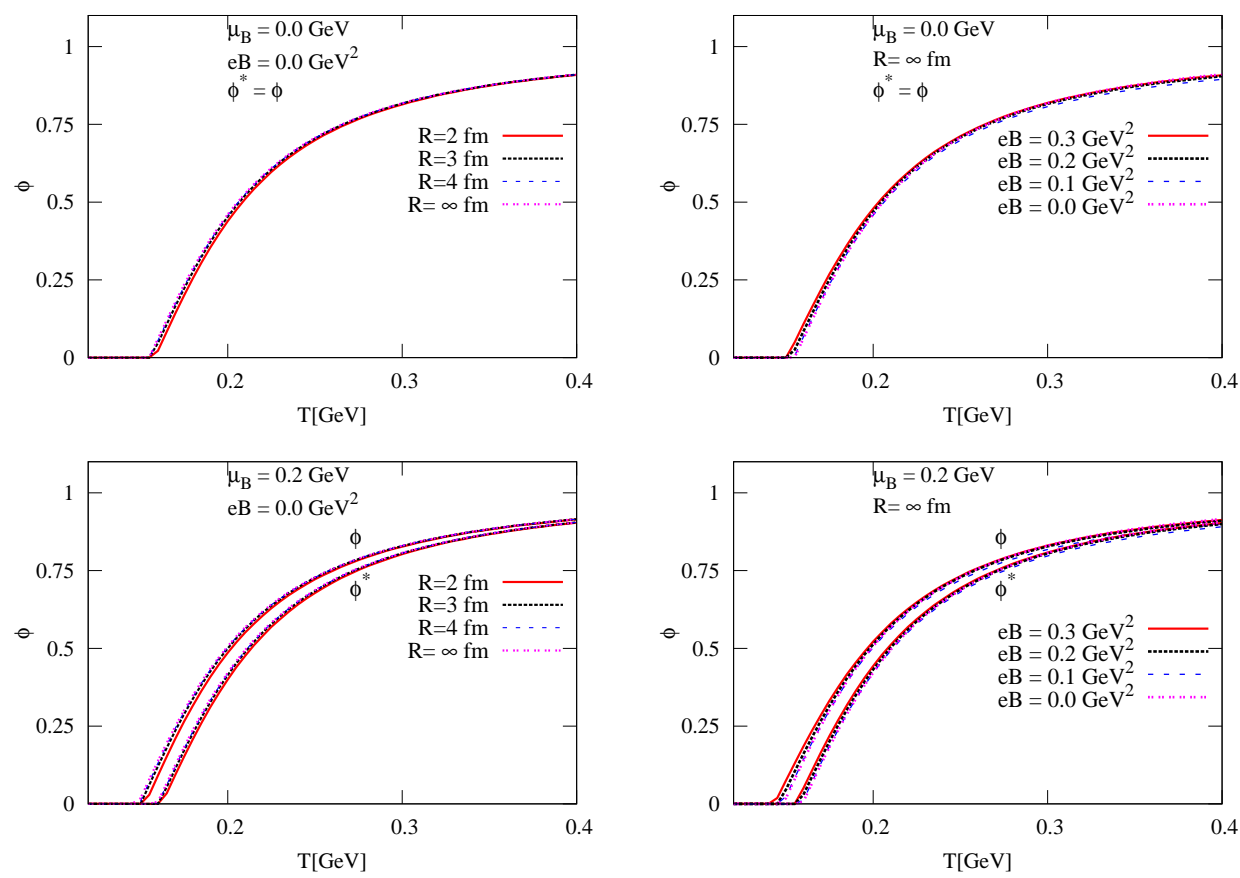

Fig. 2. (Color online) The same as in Fig.(11) but for the two Polyakov loops.

and the magnetic field strength. For $\mu_{B}=0.2 \mathrm{GeV}$, a weak dependence, with trends similar to those in the bottom panels of Fig. 1, can be observed.

\section{B. Thermodynamics properties}

The pressure $P$ is easily obtained from the grand potential as,

$$
P=-\Omega\left(T, e B, \mu_{f}\right),
$$

where Eq. (12) expresses the [explicit] dependence of the pressure on the temperature, chemical potential, system volume and the magnetic field strength. Coupled with the energy density $\epsilon$, this pressure can also be used to obtain the trace anomaly $\Delta=\epsilon-3 P$ and the equation of state $P / \epsilon$, and to study the influence of finite volume and magnetic field effects on them. Before discussing these effects, it is instructive to compare the values for $P, \Delta$ and $P / \epsilon$ obtained from our PLSM calculations (for $\mu_{B}=0$ and $e B=0 \mathrm{GeV}^{2}$ ), to similar results from LQCD calculations [46, 54]. Such comparisons are shown in Fig. 3, they indicate reasonable agreement between the PLSM and LQCD results for the model parameters summarized in Table [1.

Figure 4 shows the temperature dependence of the normalized pressure for different vol- 

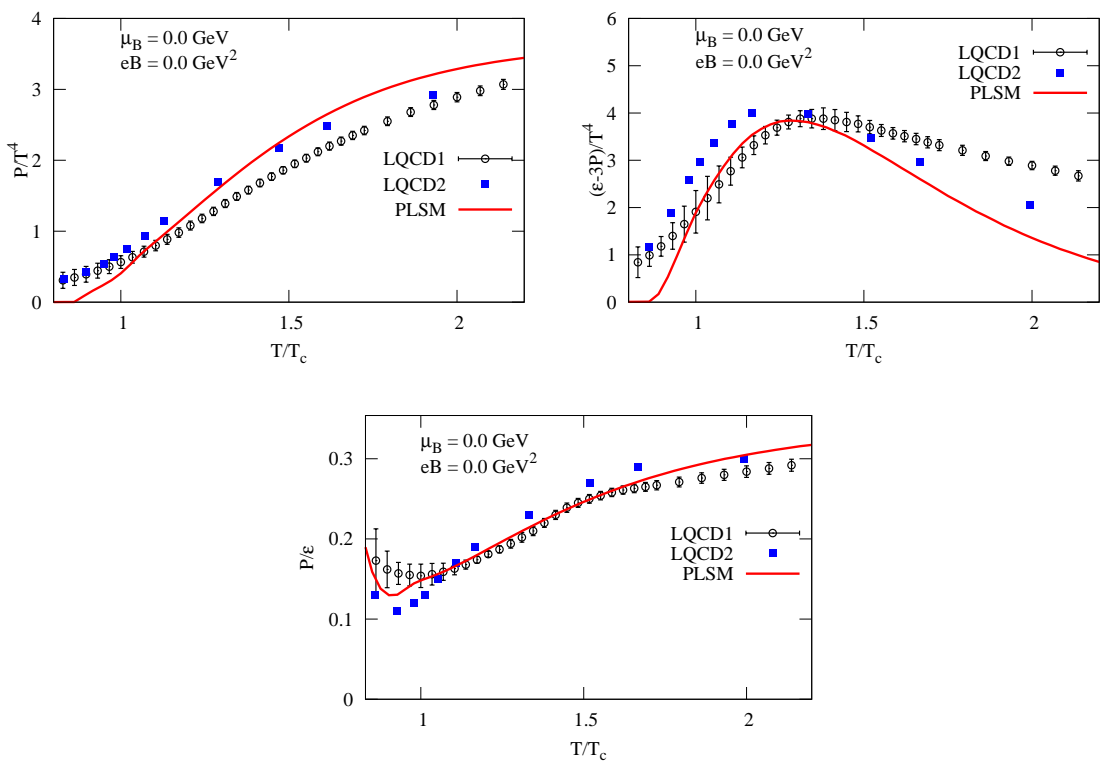

Fig. 3. (Color online) Comparison of the PLSM pressure density (left panel), trace anomaly (middle panel) and $P / \epsilon$ (right panel) to results from LQCD. The comparisons are made for $\mu_{B}=0$; the solid lines indicate PLSM results and the open and closed symbols indicate LQCD results from Refs. [46] and [54] respectively.

ume and magnetic field selections for two values of $\mu_{B}$. The left panels indicate an increase of the normalized pressure with volume which quickly trends towards the infinite volume value. The right panels show that the normalized pressure also increase with magnetic field strength, but do not trend toward a saturation value for the range of magnetic field strengths studied.

Figure 5 shows the thermal behavior of the normalized trace anomaly for several volume and magnetic field selections for the previously used values of $\mu_{B}$. The left panels indicate that the normalized trace anomaly is insensitive to volume changes for $T \lesssim 0.2 \mathrm{GeV}$. For larger temperatures, the normalized trace anomaly decrease with increasing volume and quickly saturates to the infinite volume value. The right panels indicate a similar dependence of the normalized trace anomaly as a function of magnetic field strength for the full range of temperatures studied. That is, they show a decrease in magnitude with increasing magnetic field strength over the full temperature range.

The left panels of Fig. [6 show that $P / \epsilon$ is relatively insensitive to the volume at low temperatures. For higher temperatures, it shows an increase $(P / \epsilon$ gets softer) with volume which quickly saturates to the infinite volume value especially for the highest temperatures. 

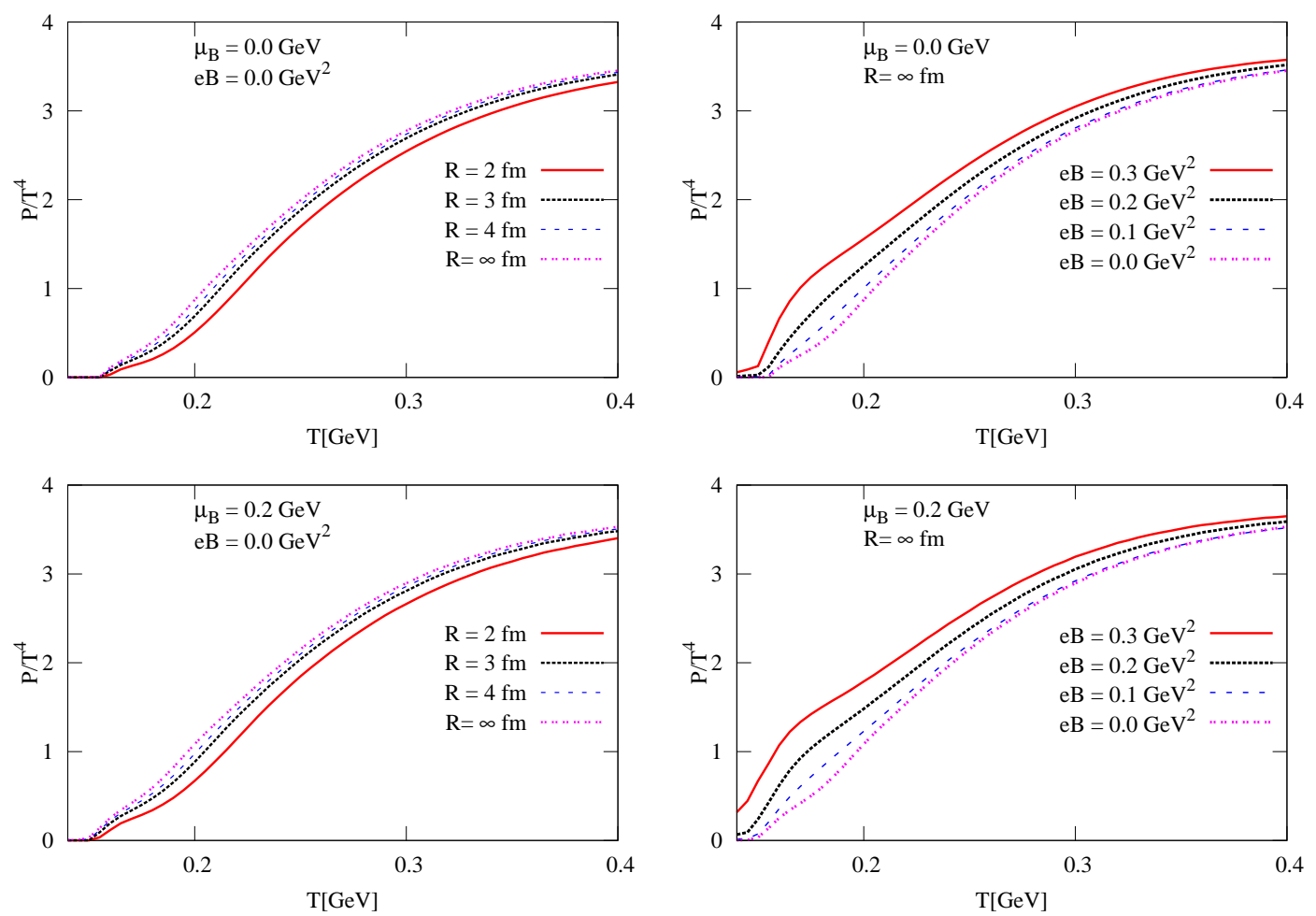

Fig. 4. (Color online) Temperature dependence of the normalized pressure, for several volume selections with $e B=0$ (left panels) and for several $e B$ selections at infinite volume (right panels). Results are shown for $\mu_{B}=0 \mathrm{GeV}$ (top panels) and $\mu_{B}=0.2 \mathrm{GeV}$ (bottom panels). .

The right panels of Fig. 6] also indicate an increase of $P / \epsilon$ with magnetic field strength, especially at low temperatures. Here, the magnitudes and trends are in stark contrast to those for the volume dependencies shown in the left panels.

\section{QCD phase diagram}

The PLSM has two chiral order-parameters (the strange and non strange chiral condensates) which reflect the chiral phase transitions. To investigate finite volume and magnetic field effects on the $S U(3) 2+1$ PLSM chiral phase transition, we use the normalized netdifference condensate $\Delta_{q, s}(T)$ as,

$$
\Delta_{q, s}(T)=\frac{\sigma_{x}-\frac{m_{q}}{m_{s}} \sigma_{y}}{\sigma_{x 0}-\frac{m_{q}}{m_{s}} \sigma_{y 0}},
$$

where $m_{q}\left(m_{s}\right)$ are non-strange (strange) quark masses. $\Delta_{q, s}(T)$ reflect the PLSM chiral phase transition. Fig. 7 shows that the chiral phase transition is influenced by the effects 

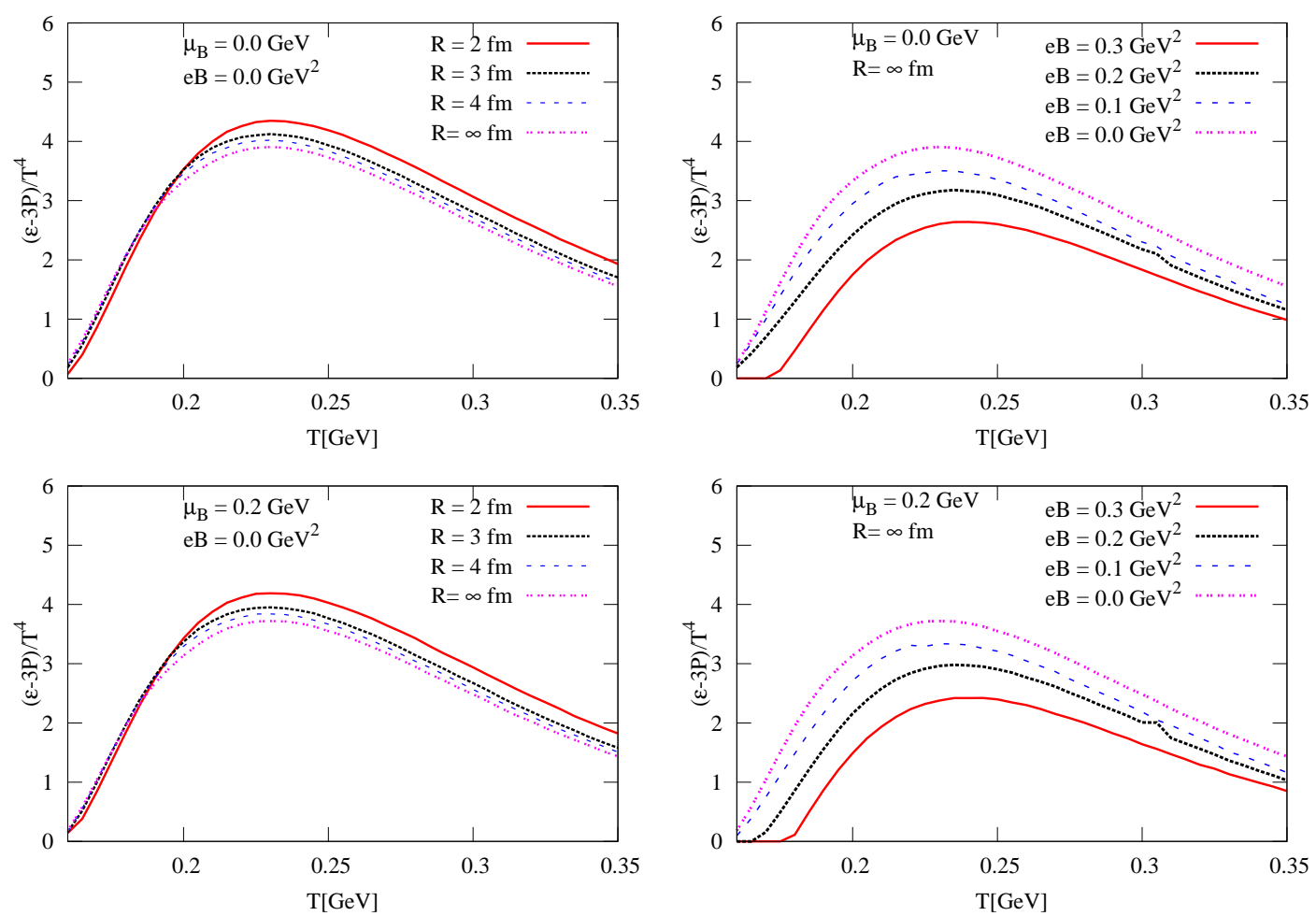

Fig. 5. (Color online) Temperature dependence of the normalized trace anomaly for several volume selections with $e B=0 \mathrm{GeV}^{2}$ (left panels) and for several $e B$ selections at infinite volume (right panels). Results are shown for $\mu_{B}=0 \mathrm{GeV}$ (top panels) and $\mu_{B}=0.2 \mathrm{GeV}$ (bottom panels).

of a finite volume and the magnetic field. The left panels indicate an increase in $\Delta_{q, s}(T)$ as the system volume is decreased. This trend contrasts with the influence of the magnetic field which results in a decrease of $\Delta_{q, s}(T)$ with increasing magnetic field strength. Thus, the effects of finite size have an opposing influence to those for the magnetic field.

The PLSM phase diagram (for a fixed volume and magnetic field strength) can be extracted with the aid of $\Delta_{q, s}(T)$. For fixed values of $R, e B$ and $\mu_{B}$, the $d \Delta_{l, s} / d T$ is deduced as a function of temperature (cf. Fig. 8). For the same baryon chemical potential, $d \Delta_{l, s} / d T$ will peak up at a characteristic point indicating the phase transition. Thus, the phase diagram can be generated by mapping such points for a broad range of baryon chemical potentials. Fig. 9 illustrates the effects of finite volume and the magnetic field on the phase diagram. The left panel shows that the phase boundary in the $\left(\mu_{B}, T\right)$-plane of the PLSM phase-diagram, increases with decreasing system volume, i.e., both $T$ and $\mu_{B}$ increase as we decrease the system volume. A similar volume dependence has been reported Ref. [38]. The right panel shows that the effects of the magnetic field contrasts with the finite vol- 

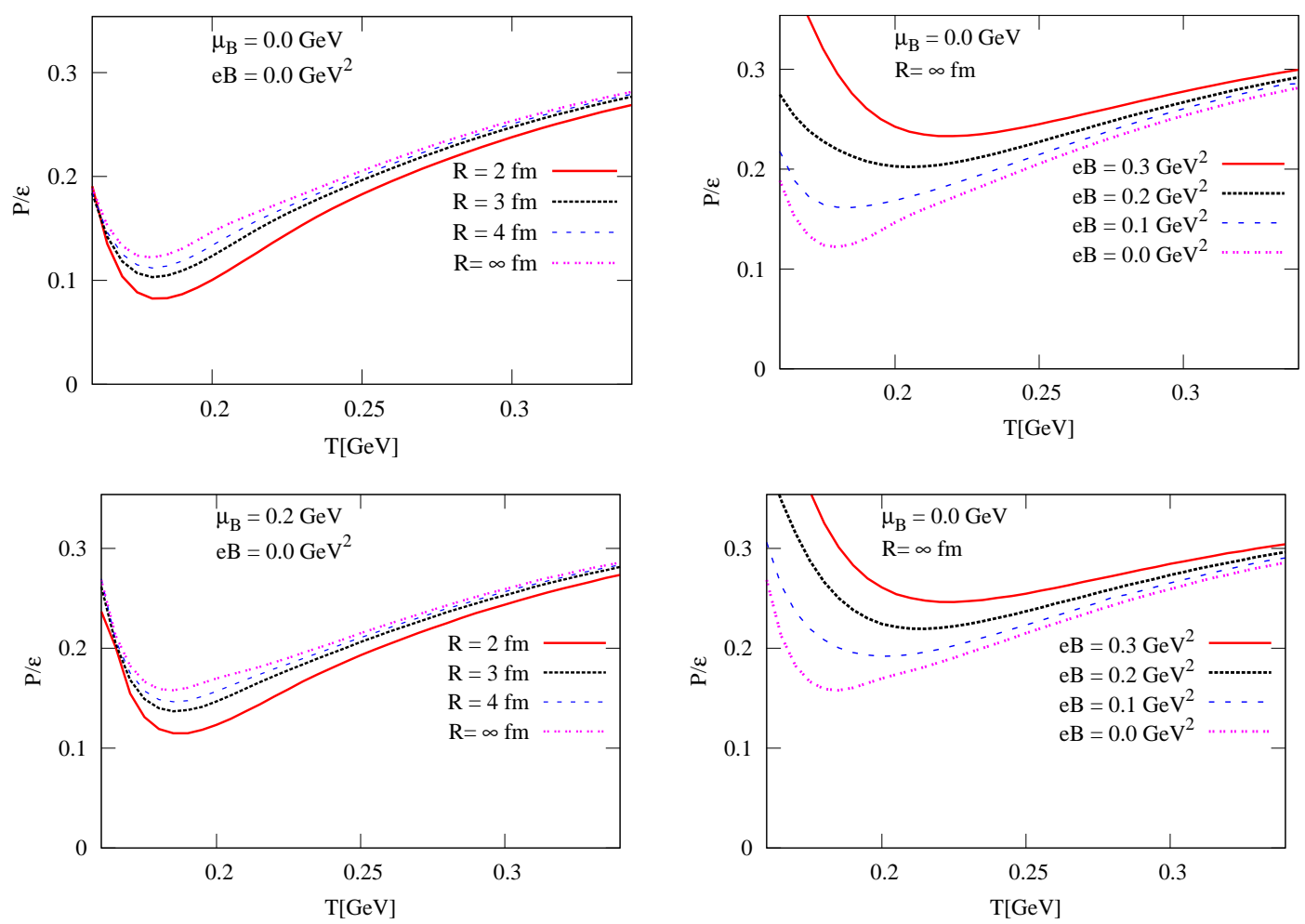

Fig. 6. (Color online) $P / \epsilon$ vs. $T$ for several volume selections with $e B=0 \mathrm{GeV}^{2}$ (left panels) and for several $e B$ selections at infinite volume (right panels). Results are shown for $\mu_{B}=0 \mathrm{GeV}$ (top panels) and $\mu_{B}=0.2 \mathrm{GeV}$ (bottom panels).

ume effects. That is, the phase boundary shifts to lower values in the $\left(\mu_{B}, T\right)$-plane as the magnetic field strength is increased.

\section{CONCLUSIONS}

In this work we have used the $2+1 S U(3)$ PLSM framework to investigate the properties of the QCD medium produced at finite volume and finite magnetic fields in heavy ion collisions. This model framework gives several thermodynamic quantities which compare well with those obtained in LQCD calculations for vanishing $e B$ and $\mu_{B}$. The PLSM calculations indicate that the confinement order parameters or Polyakov loops ( $\phi$ and $\left.\phi^{*}\right)$ are relatively insensitive to changes in the volume and the magnetic field strength. This contrasts with the chiral condensates which show a much larger sensitivity, albeit with much larger sensitivity for the non-strange chiral order parameter. Both chiral condensates are found to increase with decreasing system volume, but decrease with increasing magnetic field strength. 

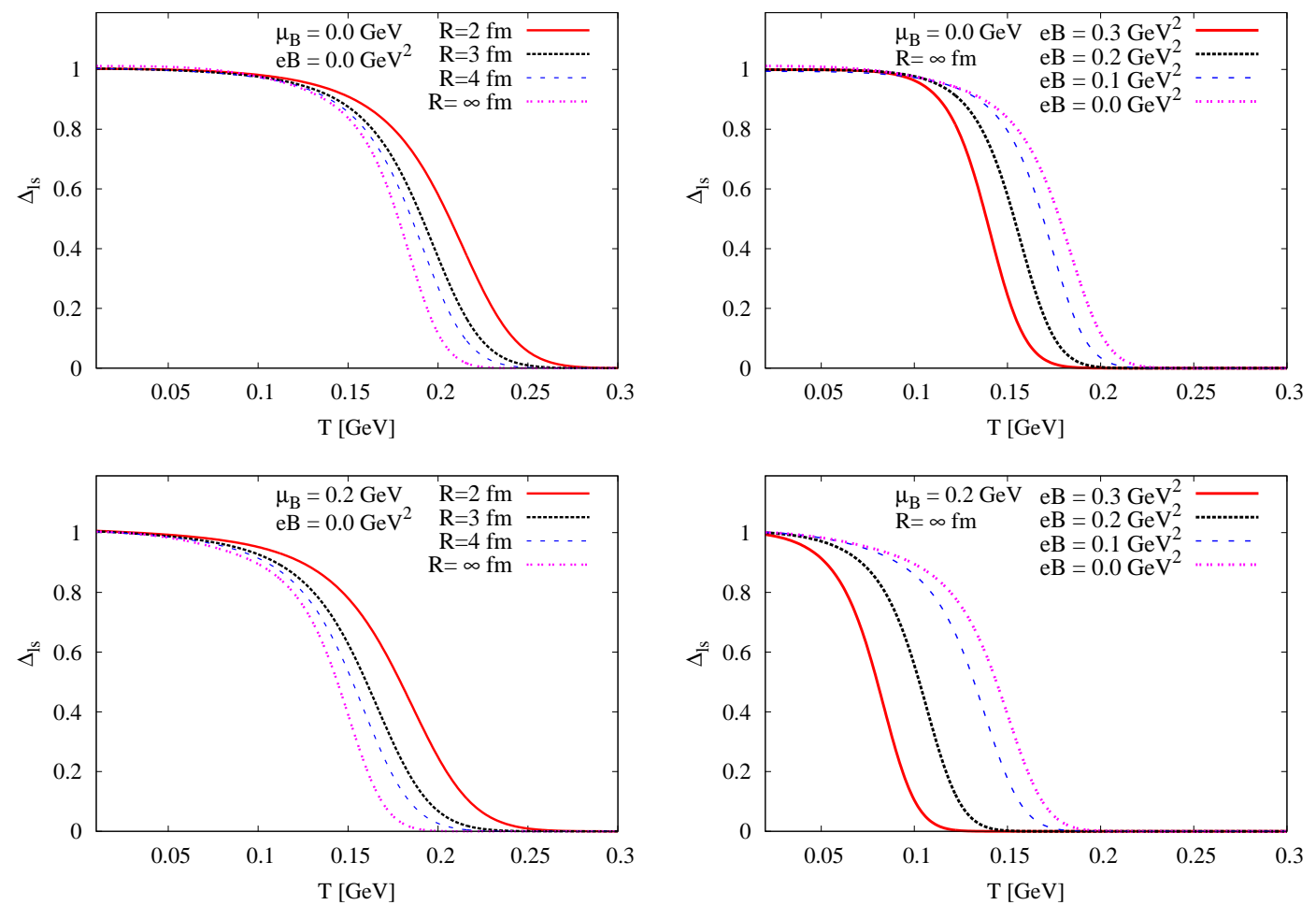

Fig. 7. (Color online) Temperature dependence of the net-difference condensates $\Delta_{l s}$, for several volume selections with $e B=0$ (left panels) and for several $e B$ selections at infinite volume (right panels). The results are shown for $\mu_{B}=0 \mathrm{GeV}$ (top panels) and $\mu_{B}=0.2 \mathrm{GeV}$ (bottom panels).

The PLSM calculations also indicate that several thermodynamic quantities $(P, \Delta$ and $P(\epsilon)$ are significantly influenced by finite volume and finite magnetic field effects. Our combined study of PLSM thermodynamics and the chiral order parameters, suggests that the quark-hadron phase boundary is shifted to higher values of $\mu_{B}$ and $T$ with decreasing system volume, and to lower values of $\mu_{B}$ and $T$ with increasing magnetic field strength. Thus, the effect of a finite volume on the phase boundary is opposite to that for a finite magnetic field. Additional studies geared at the influence of a finite volume and a finite magnetic field on the location of the critical end point will be discussed in a future work. 

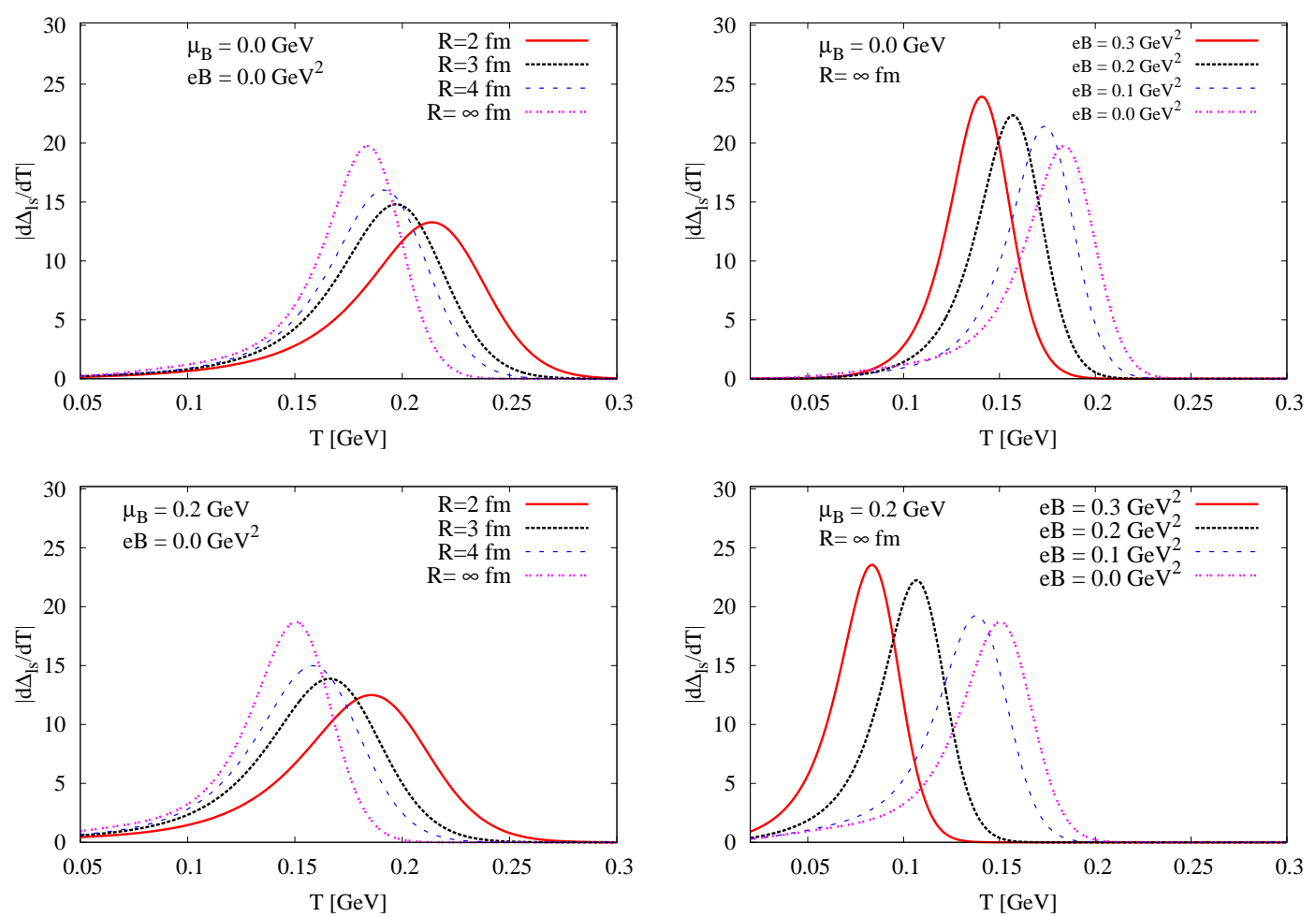

Fig. 8. (Color online) Same as in Fig.(7) but for $d \Delta_{l, s} / d T$.
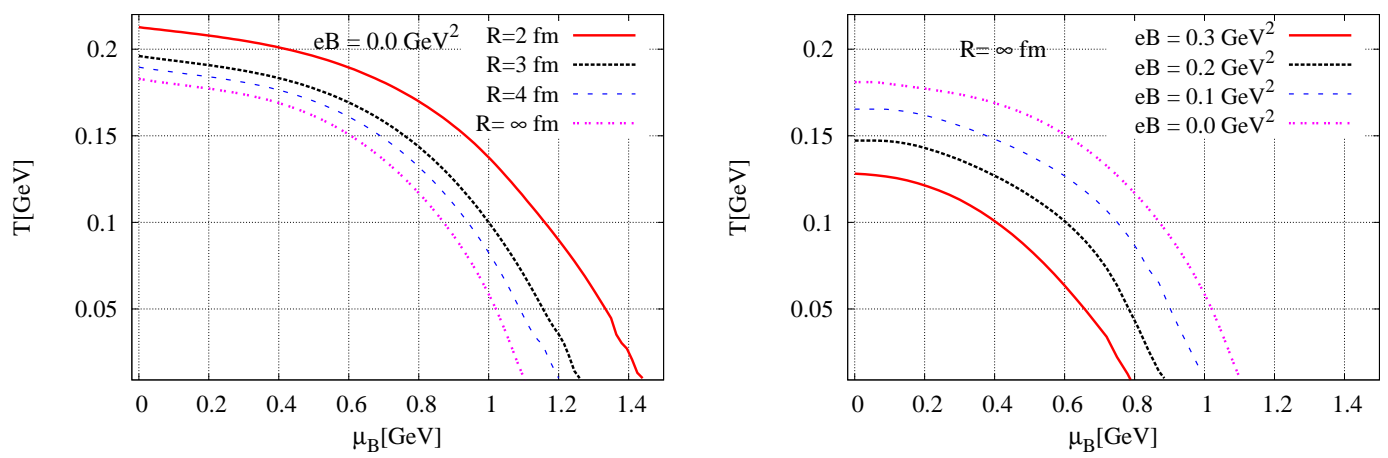

Fig. 9. (Color online) Chiral phase diagram for (i) different volumes selections for $e B=0 \mathrm{GeV}^{2}$ (left panel) and (ii) different $e B$ selections for $R=\infty$ fm (right panel). 
[1] Experimental highlights of the RHIC program, P. Fachini, AIP Conf.Proc. 857 62-75 (2006). hep-ex/0605102

[2] The SPS heavy ion programme, Helmut Satz, Phys.Rept. 403-404 33-50 (2004).

[3] Physics programme of ALICE experiment, K. Safarik, Nucl.Phys. A 749 229-242 (2005).

[4] FAIR and its experimental program W.F. Henning, J.Phys. G 34 S551-S557 (2007).

[5] Heavy-ion program at NICA/MPD at JINR Alexander Sorin, Vladimir Kekelidze, Alexander Kovalenko, Richard Lednicky, Igor Meshkov, Grigory Trubnikov, Nucl.Phys. A 855 510-513 (2011).

[6] Y. Aoki, G. Endrodi, Z. Fodor, S. D. Katz and K. K. Szabo, Nature 443, 675 (2006).

[7] F. R. Brown et al., Phys. Rev. Lett. 65, 2491 (1990).

[8] S. Ejiri, Phys. Rev. D 78, 074507 (2008).

[9] R. D. Pisarski and F. Wilczek, Phys. Rev. D 29, 338 (1984).

[10] M. Asakawa and K. Yazaki, Nucl. Phys. A 504, 668 (1989).

[11] M. A. Halasz, A. D. Jackson, R. E. Shrock, M. A. Stephanov and J. J. M. Verbaarschot, Phys. Rev. D 58, 096007 (1998).

[12] Y. Hatta and T. Ikeda, Phys. Rev. D 67, 014028 (2003).

[13] E. S. Bowman and J. I. Kapusta, Phys. Rev. C 79, 015202 (2009).

[14] Roy A. Lacey, Phys.Rev.Lett. 11414 (2015)

[15] Fraga, E. S., and L. F. Palhares (2012), Phys. Rev.D 86, 016008.

[16] Boomsma, J. K., and D. Boer (2009), Phys.Rev. D 80, 034019.

[17] Boomsma, J. K., and D. Boer (2010), Phys.Rev. D 81, 074005.

[18] Skokov, V., B. Friman, E. Nakano, K. Redlich, and B.-J. Schaefer (2010), Phys. Rev. D 82, 034029.

[19] Skokov, V., A. Y. Illarionov, and V. Toneev (2009), Int. J. Mod.Phys. A 24, 5925.

[20] Son, D. T., and M. A. Stephanov (2001), Phys. Rev.Lett. 86, 592.

[21] Gatto, R., and M. Ruggieri (2010), Phys. Rev. D 82,054027. Gatto, R., and M. Ruggieri (2011), Phys. Rev. D 83,034016.

[22] Mizher, A. J., M. N. Chernodub, and E. S. Fraga.(2010), Phys. Rev. D 82, 105016.

[23] DElia, M., S. Mukherjee, and F. Sanfilippo (2010),Phys. Rev. D. 82, 051501. 
[24] Jens O. Andersen, William R. Naylor, Anders Tranberg, [arXiv:1411.7176 [hep-lat]].

[25] A. E. Ferdinand and M. E. Fisher, Phys. Rev. 185, 832 (1969); M. E. Fisher and M. N. Barber, Phys. Rev. Lett. 28, 1516 (1972). M. N. Barber, in 'Phase Transitions and Critical Phenomenon', C. Domb and J. L. Lebowitz (Eds.), Academic Press (Pub.), 8, 146 (1987); J. L. Cardy, Scaling and Renormalization in Statistical Physics, (Cambridge, New York, 1996); D. Amit, Field Theory; The Renormalization Group and Critical Phenomena, (World Scientific, 2005).

[26] L. M. Abreu, M. Gomes and A. J. da Silva, Phys. Lett. B 642, 551 (2006).

[27] L. F. Palhares, E. S. Fraga and T. Kodama, J. Phys. G 38, 085101 (2011).

[28] E. S. Fraga, L. F. Palhares and P. Sorensen, Phys. Rev. C 84, 011903(R) (2011).

[29] A. Bhattacharyya, P. Deb, S. K. Ghosh, R. Ray and S. Sur, Phys. Rev. D 87, 054009 (2013). A. Bhattacharyya, R. Ray, S. Sur, Phys. Rev. D 91, 051501 (2015).

[30] G.Endrődi , JHEP 1304, 023 (2013).

[31] S.P. Klevansky, Rev. Mod. Phys. 64, 649 (1992).

[32] I.A. Shushpanov and A.V. Smilga, Phys. Lett. B 402, 351(1997).

[33] N.O. Agasian and I.A. Shushpanov, Phys. Lett. B 472, 143 (2000).

[34] T.D. Cohen, D.A. McGady, and E.S. Werbos, Phys. Rev. C 76, 055201 (2007).

[35] D. Kabat, K.M. Lee, and E. Weinberg, Phys. Rev. D 66, 014004 (2002).

[36] M. Ruggieri, M. Tachibana and V. Greco, JHEP. 1307, 165, (2013).

[37] Abdel Nasser Tawfik and Niseem Magdy, Phys.Rev. C 90015204 (2014).

[38] L. F. Palhares, E. S. Fraga and T. Kodama, J. Phys.G 38, 085101,2011.

[39] Ferreira, M., P. Costa, O. Lourenco, T. Frederico,and C. Providencia (2014a), Phys. Rev. D $\mathbf{8 9}, 116011$.

[40] A. Tawfik, N. Magdy and A. Diab, Phys. Rev. C 89, 055210 (2014).

[41] B.-J. Schaefer and M. Wagner, Nucl. Phys. 62, 381 (2009).

[42] Hong Mao, Jinshuang Jin and Mei Huang, J. Phys. G37, 035001 (2010).

[43] K. Fukushima, Phys. Rev. D 77114028 (2008).

[44] B. J. Schaefer and M. Wagner, Phys. Rev. D 79, 014018 (2009).

[45] J. T. Lenaghan, D. H. Rischke and J. Schaffner-Bielich, Phys. Rev. D 62, 085008 (2000).

[46] Szabolcs Borsányi, et al, "Full result for the QCD equation of state with 2+1 flavors", Phys.Lett. B 730 99-104 (2014). 
[47] Lisa M. Haas, Rainer Stiele, Jens Braun, Jan M. Pawlowski, Juergen Schaffner-Bielich, . Phys. Rev. D 87, 076004 (2013).

[48] B.Jochen, Schaefer, M.Wagner, J.Wambach, "QCD thermodynamics with effective models", PoS CPOD2009:017,(2009).

[49] Peter Kovács, Gyögy Wolf, "Chiral phase transition scenarios from the vector meson extended Polyakov quark meson model", Acta Phys.Polon.Supp. 8335 (2015).

[50] Peter Kovács, Zsolt Szép, Gyögy Wolf, ” Existence of the critical endpoint in the vector meson extended linear sigma model", 1601.05291 [hep-lat].

[51] Wei-jie Fu , Phys. Rev. D 88, 014009 (2013).

[52] Abhijit Bhattacharyya, Rajarshi Ray, Subhasis Samanta and Subrata Sur, 1502.00889 [hep-ph].

[53] Bernd-Jochen Schaefer and Mathias Wagner, Phys.Rev. D79, 014018 (2009).

[54] Szabolcs Borsányi, Gergely Endrődi, Zoltan Fodor, Antal Jakovác, et.al, "The QCD equation of state with dynamical quarks", JHEP 1011077 (2010). 